\title{
Neue Untersuchungen über Metallnebel. Nr. I. Über Bleinebel in Bleichlorid.
}

\author{
Von \\ Richard Lorenz und W. Eitel. \\ Mit 5 Figuren im Text und 3 Tafeln.
}

Über die von R. LoRENz in den Jahren 1898-1899 entdeckte Erscheinung der „Metallnebel" ist wiederholt berichtet worden. ${ }^{1}$ Wirft man in ein geschmolzenes Salz, z. B. Bleichlorid, ein Stückchen metallisches Blei ein, so bemerkt man, wie das Metall, nachdem es unter der Schmelze zu einem quecksilberglänzenden Tropfen geschmolzen ist, plötzlich eine dichte, schwarzbraune Wolke ausstöBt, die sich in der Flüssigkeit verteilt und diese intensiv färbt. Diese Färbung verwandelt die ursprünglich klare und durchsichtige Schmelze bald in eine tiefdunkle Flüssigkeit, die in intensivem durchfallenden Licht in geringer Dicke mit brauner Farbe noch durchsichtig ist. Die Erscheinung, welche auch bei der Elektrolyse der geschmolzenen Salze an der Kathode beobachtet wird und bei dem elektrochemischen Verhalten von Salzschmelzen (Stromausbeute, Polarisation, Reststrom usw.) eine wichtige Rolle spielt, ist schon öfters photographiert worden. Eine neuere, besonders anschauliche Aufnahme, welche uns gelungen ist, ist in Fig. 1, Tafel VI wiedergegeben. Man

1 Eine Zusammenstellung der Literatur über Metallnebel findet sich in R. Lorenz, Elektrolyse der geschmolzenen Salze, Bd. 2, (Halle 1905), S. 40 und folgende. Ferner R. Lorenz und F. KadFuER, Elektrochemie geschmolzener Salze (Bredias Handb. d. angew. phys. Chem., Bd. 2, Abt. 1, (Leipzig 1909), S. 22 und folgende. Siehe ferner R. Lorenz, Otтo WaLLach-Festschrift, (Göttingen 1909), S. 513. - R. Lorenz, "Pyrosole", I. M. van Bemmelen-Festschrift, (Helder 1910), S. 395. - R. Lorenz, G. v. Hevesp u. E. WolfF, Beiträge zur Kenntnis der Natur der Metallnebel in Schmelzflüssen, Zeitschr. phys. Chem. 76 (1911), 732. - R. Lorenz und A. Liebmann, Bestimmung der Oberflächenspannung von geschmolzenem Blei gegen geschmolzene Mischungen von Bleichlorid und Kaliumehlorid, Zeitschr. phys. Chem. 83 (1913), 459. - A. H. W. Atrn, Elektrizitätsleitung in Mischungen von Metallen und ihren Salzen, Zeitschr. phys. Chem. 73 (1910), 578. 
erkennt das raketenartige AufschieBen der Nebel deutlich auf dieser Aufnahme.

In dem unteren Teile des Rohres erkennt man auf der Photographie die eigentümlich gestalteten wolkenartigen Gebilde. Ferner ist in der Mitte des helldurchleuchteten Teiles des Rohres ein kometenartiges Gebilde zu erkennen, welches an seinem oberen Ende einen dunklen Kern besitzt und dessen Schweif bis zum Meniskus des geschmolzenen Bleis zurückreicht. Die rechts von diesem Schweif befindliche Wolke ist eben in ähnlicher Weise im Aufsteigen begriffen. Die dunklen Schattenpartien bei der Einschnürung des Rohres rühren von unvermeidlichen Reflexen bei der Aufnahme des stark lichtbrechenden Bleichloridschmelzflusses im durchfallenden Lichte her und sind nicht mit den Nebeln zu verwechseln. Die senkrecht verlaufenden geraden Linien rühren aus dem Glase her.

Was die Natur der Metallnebel anbelangt, so wird im folgenden gezeigt, dab es sich in Übereinstimmung mit der von $R$. Lonenz wiederholt ausgesprochenen Ansicht um eine Erscheinung aus dem Gebiete der Kolloidchemie handelt. Leider sind die technischen Schwierigkeiten der Beobachtung des kolloidalen Phänomens im SchmelzfluB bei hoher Temperatur immer noch nicht endgültig überwunden. Hingegen ist es uns nunmehr gelungen, den Nachweis des kolloidalen Zustandes in den erstarrten Schmelzen zu erbringen.

\section{Darstellung von reinem, optisch leeren Bleichlorid.}

Reinstes käufliches Bleichlorid von E. Merck-Darmstadt wurde in Röhren von schwerschmelzbarem Kaliglas (Bombenröhren) im elektrischen Röhrenofen mit Nickeldrahtheizung eingeschmolzen (je $100 \mathrm{~g}$ ) und hierauf mit einem Strom von einem Gemisch von scharf getrocknetem Chlorgas und trockenem Chlorwasserstoff längere Zeit (1-2 Ștunden) behandelt (siehe Fig. 1). Die Geschwindigkeit der Gasströme wurde durch Blasenzählung in den Waschflaschen so bemessen, daB ungefähr gleiche Volumina der Gase sich mischten. Der Zweck dieser Behandlung besteht darin, erstens etwa vorhandene Spuren von Wasser und Oxychlorid bzw. Hydroxychlorid (Hydrolyse in der Bleichloridschmelze ${ }^{1}$ ) zu entfernen, zweitens etwa schon vor-

1 Auf das Vorbandensein einer Hydrolyse bei geschmolzenen Salzen ist wiederholt aufmerksam gemacht worden; vgl. z. B. R. LonENz, Elektrolyse der gesehmolzenen Salze, Bd. 2, (Halle 1905), S. 44. - R. Lorenz und F. KaUfler, Elektrochemie geschmolzener Salze, (Bredies Handb. d. angew. phys. Chemie, Bd. 2, Abt. 1, Leipzig 1909), S. 26 u. folg. Die Hydrolyse wird auch stets 
handene Bleinebel in der Schmelze zu vernichten. Die weitere Aufgabe bestand darin, die so erhaltene, völlig reine Schmelze von Bleichlorid in wohlausgebildeten Kristallen erstarren zu lassen. In runden Kaliglasröhren wurden in der Regel keine schönen Kristallisationen erzielt, weil sich hierbei die von Tammann beschriebene Erscheinung einstellt, daß die Kristalle sich durch die gleichmäBige Wärmeabgabe der zylindrischen Röhre radial oder längs der Röhrenachse anordnen und dann zu schmal ausfallen. Wir fanden, daB

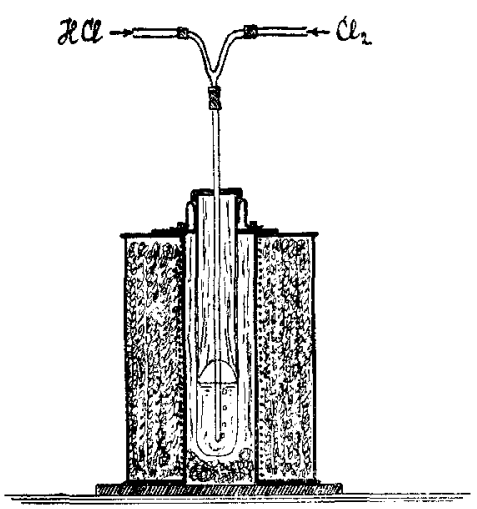

Fig. 1. Apparat zur Entfernung der Bleinebel in geschmolzenem Bleichlorid. die besten Kristalle in Röhren erhalten wurden, welche an ihrem unteren geschlossenen Ende plattgedrückt sind, so dab annähernd ein parallelepipedisches Gefäß entsteht. Die Kristalle bilden sich dann meist nach der Basisfläche und der Längsfläche aus. Das Erkalten darf nicht zu langsam vor sich gehen, vielmehr hat sich als vorteilhaft herausgestellt, nach Beendigung des Reinigungsprozesses die Röhren aus dem Ofen herauszunehmen und an der Luft erkalten zu lassen. Um die sehr leicht spaltbaren Kristalle nicht zu zertrümmern wurden die Röhren behutsam am oberen und unteren Ende des plattgedrückten Teiles angefeilt und abgesprengt, worauf die erstarrte Schmelze sich fast immer in kompakter Form glatt herausnehmen lieB. Sie stellt eine vollkommen klare, weiße Kristallmasse dar (nicht etwa gelb oder gelblich!), aus der sich durch vorsichtiges Abspalten mit dem Taschenmesser leicht schöne Einzelkristalle gewinnen lassen, welche vollständig wasserklar und diamantglänzend und unter dem Ultramikroskop optiseh leer sind.

\section{Beschreibung der Kristalle.}

Da Bleichloridkristalle aus dem Schmelzflusse noch nicht beschrieben sind, ${ }^{1}$ sollen hier einzelne diesbezügliche Angaben gemacht

von Tн. W. Richards bei seinen Atomgewichtsbestimmungen aus Metallchloriden berücksichtigt, und ist auch von Le Blavo beobachtet und bestätigt worden, der den treffenden Ausdruck "hygroskopische Schmelzen" geprägt hat.

1 Vgl. P. Gвотн, Chemische Kristallographie, Leipzig 1906, S. 219. 
werden. Die Kristallformen sind sehr einfach. In der Regel beobachtet man nur die Pinakoide (001) (Basis) und (010) (Längstläche), sowie die meist undeutlich ausgebildete Pyramide (121), daneben böchstens noch das Doma (012), welches einmal beobachtet wurde. Die Symmetrie entspricht durchaus dem rhombisch-bipyramidalen Verhältnis, wie man dies an künstlichen Kristallen aus wässerigen Lösungen seit geraumer Zeit kennt. Was die Kohäsionseigenschaften betrifft, so fällt vor allem die Spaltbarkeit nach (001) auf. Sie ist

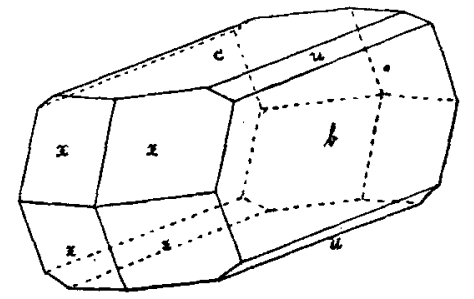

Fig. 2. Kristall von Bleichlorid aus dem Schmelzen. $x\{121\}, b\{010\}, c\{001\}, u\{012\}$.

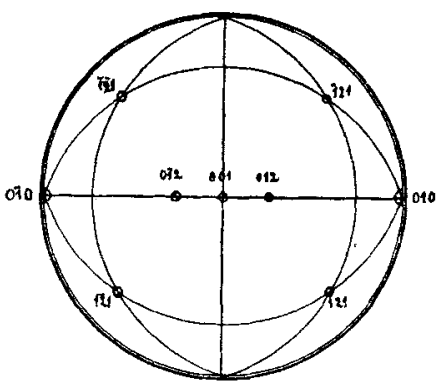

Fig. 3. Stereographische Projektion des Bleichloridkristalles in Fig. 3.

als vorzüglich zu bezeichnen. Zum Unterschied von den aus Lösungen erhaltenen Kristallen ist aber auch eine, wenn auch unvollkommene Spaltbarkeit nach dem Pinakoid (010) zu bemerken. Die Härte ist sehr gering, sie dürfte etwa der des Steinsalzes gleichen. Das spezifische Gewicht beträgt bei $17^{\circ} \mathrm{C} 5.840$. Die Kristalle sind, wie erwähnt, völlig wasserklar und zeigen starken Diamantglanz. Der mittlere Brechungsexponent ergab sich $\mathrm{zu}>2.10$ und $<2.30$. Die Doppelbrechung ist sehr stark und zwar mit positivem Charakter, sie findet sich in der Literatur für Kristalle aus wässeriger Lösung zu 0.060 angegeben. Die Achsenebene verläuft parallel (010), der Achsenwinkel beträgt an Kristallen aus Lösung gemessen nach P. GROTH $2 V=66^{\circ} 12^{\prime}$. Fig. 2 und 3 stellt einen derartigen Kristall in idealisierter Form dar. Die ultramikroskopische Untersuchung, über welche weiter unten berichtet wird, ergab bei diesen reinen Kristallen völlige Abwesenheit des Tyndallkegels, ${ }^{2}$ sie sind also vollkommen optisch leer. Nur die

1 P. Grotr, Chemische Kristallographie, (Leipzig 1906), S. 220.

1 Unter "Tyndallkegel" verstehen wir in üblicher, wenn auch nicht streng richtiger Weise den sichtbaren Beleuchtungskegel, der in kolloidalen Medien entsteht.

Z. anorg. Chem. Bd. 91. 
Spaltrisse nach (001) und (010) zeigen sich meistens in Gestalt scharf begrenzter blendend heller Linien, die sich unter $90^{\circ}$ trefien (vgl. Tafel VIII, Fig. 13).

Zuweilen bemerkt man auch Einschlüsse von Gasbläschen, welche wegen der starken Doppelbrecbung der Kristalle doppelt erscheinen.

\section{Erzeugung der Bleinebel.}

Reinstes optisch leeres Bleichlorid wurde im elektrischen Ofen in einem Kaliglasrohr von der beschriebenen Form eingeschmolzen und die Temperatur auf etwa $100-150^{\circ}$ über den Schmelzpunkt des Bleichlorids gesteigert. Hierauf wirft man einige Schnitzel dünnen vorher blank geschabten Bleibleches in die Schmelze und läßt sie einige Zeit (ca. 5 Minuten) bei der höheren Temperatur einwirken. Jetzt wird das Rohr aus dem Ofen genommen und die nun ganz schwarz erscheinende Schmelze erstarren gelassen. Die wie früher gewonnene Kristallmasse ist grauglänzend, die einzelnen, aus denselben abgespaltenen Kristalle erscheinen jedoch immer noch durchsichtig, wenn auch nicht mehr wasserklar. Die Eigenschaften sind dieselben wie oben beschrieben.

\section{Ultramikroskopische Untersnchung.}

Behufs ultramikroskopischer Untersuchung spaltet man einen Kristall möglichst glatt und poliert die erhaltene Fläche auf weichem Hirschleder bis auf Hochglanz. Dann verfährt man ebenso mit einer zweiten dazu senkrechten Fläche. Unter günstigen Umständen konnten wir jedoch auch eine dieser Flächen sparen, indem wir die glatten Absonderungsflächen der Kristalle von der Wandung des GefäBes benutzten. Um diese kleinen Kristälichen im Spaltultramikroskop nach ZsigmoNDY und SIEDENTOPF untersuchen zu können, wurde ein besonderer kleiner Apparat konstruiert, der zum Halten und Einstellen des Präparates geeignet ist. Er besteht (siehe Fig. 4) aus einem Tischchen $T$, das an einem Drahtstift $D$ in einer am Ultramikroskop angebrachten Klemmschraube $K$ eingespannt werden kann. Unterhalb $T$ ist ein Schlitten $S c h l$, der auf zwei Schrauben $S_{1} S_{2}$ gleitet, angebracht, der mittels des Handgriffes $G$ senkrecht zur Achse des Beleuchtungskegels verschoben werden kann. Auf diesem Schlitten wird in einer federnden Hülse $H$ der zum Tragen des Kristalles bestimmte Messingstift $S t$ eingesteckt und dann mit einem Klümpchen Plastolin der Kristall $K r$ auf $S t$ gekittet. Durch Verstellen des Stiftes $D$ und des Schlittens Schl. läBt sich jede beliebige Stelle des 
Kristalles in den Lichtkegel des Ultramikroskops bringen. Der ganze zur Untersuchung verwendete ultramikroskopische Apparat ist in Fig. 5 wiedergegeben.

Das mit Bleinebeln erstarrte Bleichlorid zeigt unter dem Ultramikroskop einen überraschenden Anblick. Man erkennt ähnlich wie bei den Rubingläsern zahllose glänzende Lichtpünktchen auf tiefschwarzem Grunde, die auf die Anwesenheit einer feinverteilten Materie in den Kristallen schlieBen lassen. Aber jedes Beugungsscheibchen erscheint infolge der starken Doppelbrechung des Bleichlorids doppelt und auch die um das Scheibchen konzentrisch

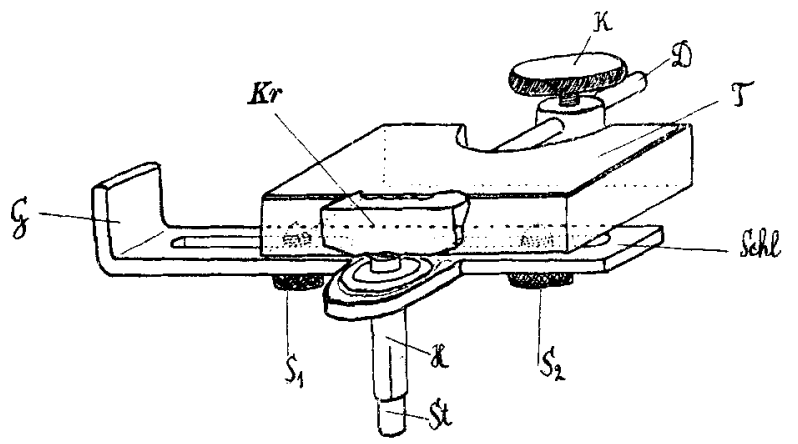

Fig. 4. Vorrichtung zur Untersuchung von Salzkristallen auf Metallnebel.

liegenden farbigen Beugungsringe zeigen dieselbe $\nabla$ erdoppelung, so $\mathrm{daB}$ ein äuBerst reizvolles Bild entsteht, das unseres Wissens noch nicht unter dem Ultramikroskop beobachtet worden ist. In Tafel VI, Fig. 2 und 3 sind solche Bilder wiedergegeben. Fig. 3 ist bei sehr schmalem Lichtkegel hergestellt. Hier erkennt man auch die Doppelbrechung besonders deutlich. Die Beugungsscheibchen selbst bestehen, bei starker VergröBerung and hoher Objektivapertur beobachtet, deutlich aus je zwei rot bzw. blau erscheinenden Einzelbildchen, eine Erscheinung, die Zsigmondy auch an den Goldrubinteilchen beobachtet hat und die auf der hohen Dispersion für verschiedene Lichtarten beruht. Ferner erkennt man bei starker Vergrößerung eigentümliche Lichtschweife um die Beugungsbildchen, welche den sogen. Lichtfiguren auf Kristallflächen überaus ähnlich sind und ähnliche Symmetrieverhältnisse wie diese zeigen. In Tafel VI, Fig. 4 sind mehrere solcher Lichtfiguren in 6000 facher VergröBerung abgebildet. ${ }^{1}$ Das von den Beugungsscheibchen ausgesendete Licht

1 Aufnahme einer Mikrophotographie bei 350 facher Vergrößerung im Ultramikroskop u. Vergrößerung des Negatips im Mikroskop auf den genanntenBetrag. 
ist vollkommen geradlinig polarisiert. Setzt man über das Mikroskopokular einen Analysator, so bemerkt man beim Drehen desselben abwechselnd Helligkeit und Auslöschung jeweils an der Hälfte der Bilder. Die Schwingungsrichtungen der geradlinig polarisierten

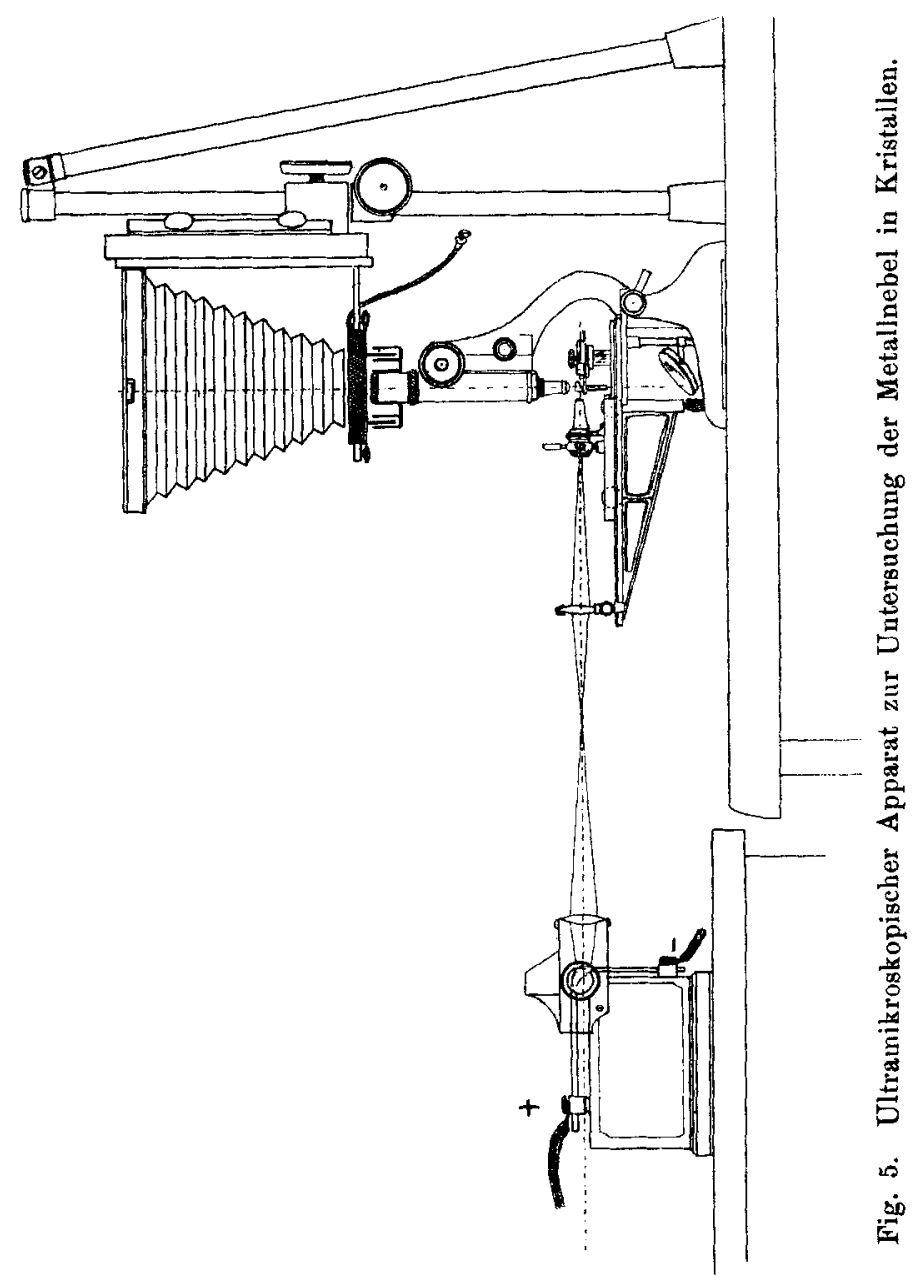

Lichtstrahlen in den beiden Beugungsbildern stehen senkrecht aufeinander. Schon SIEDENTOPF und ZsIGMONDX ${ }^{1}$ beobachteten an kolloidalen Goldteilchen in wässeriger Lösung, daB im polarisierten Lichte die von den Teilchen reflektierten Strahlen teilweise verschiedene Polarisationszustände zeigen können. Die von uns be-

I Ann. d. Phys. [4] 10 (1918), $11 \mathrm{ff}$. 
obachtete Erscheinung ist, worauf ausdrücklich hingewiesen werden soll, jedoch von der von SIEDENTOPF und ZsIGMONDY beobachteten prinzipiell verschieden. Der Unterschied besteht vor allen Dingen darin, daB in unserem Falle das Licht in dem anisotropen Medium je nach Lage der Einfallsebene zu den optischen Hauptschnitten des Kristalles in verschiedenen Richtungen verschiedene Verzögerungen erleidet, was bei den von Siedentopf und Zsiamondr beschriebenen Erscheinungen in isotropen Medien nicht der Fall sein kann. Dementsprechend macht man in unserem Falle folgende interessante Beobachtung. Geht man unter Benutzung ein und desselben Kristalles mit dem Lichtkegel bei feststehendem Mikroskoptubus in die Tiefe des Kristalles, so scheinen sich die Lichtbildchen alle voneinander zu entfernen, während sie sich bei der umgekehrten Bewegung einander nähern, weil die Dicke der von dem Licht durchlaufenen Schicht des anisotropen Mediums zunimmt bzw. abnimmt. Von Präparat zu Präparat ist die Entfernung der Beugungsbildchen bei gleicher Tiefenbeobachtung verschieden. Dies liegt einfach daran, daß die Orientierung der künstlich angebrachten Schlifffächen gegen die optischen Bezugselemente eine verschiedene ist. Theoretisch ist der Fall denkbar, daB sogar nur ein Bild gesehen werden kann, wenn nämlich eine der optischen Achsen des Kristalles mit der optischen Achse des Mikroskops zusammenfällt und senkrecht auf der Achse des Beleuchtungskegels steht. In der Tat konnten wir bei einer größeren Reihe von Präparaten und Schliffen gelegentlich dies beobachten. Wir vermuten, daB die hier beschriebene, von uns aufgefundene Erscheinung der Beugung an kolloidalen Teilchen in anisotropen Medien für die Theorie der Kristalloptik von Wichtigkeit werden kann, sobald es gelingt, dieselbe optisch messend genauer zu verfolgen.

Im AnschluB an das vorstehende sollen noch folgende Beobachtungen erwähnt werden. Das ultramikroskopische Bild gibt des öfteren AufschluB darüber, ob ein Bleichloridkristall wirklich homogen ist oder aus einem Konglomerat mehrerer verschieden orientierter kleiner Kristalle besteht. Im letzteren Falle beobachtet man im Ultramikroskop sehr feine Trennungslinien zwischen den einzelnen Kristallindividuen (Tafel VII, Fig. 5) (auch an metallnebelfreien Präparaten), und diese Trennungslinien erscheinen wie etwa vorhandene Teilchen, Einschlüsse u. dgl. im allgemeinen doppelt. Ferner erwähnen wir, daB die Teilchen selbst meist $z$ war unregelmäBig über das Gesichtsfeld verteilt sind, daB aber auch deutliche Orien- 
tierungen derselben vorkommen können. So ersieht man z. B. aus Tafel VI, Fig. 3, daB in der Nähe der durch die Mitte des Bildes verlaufenden Trennungslinie der beiden Kristallindividuen nicht ein einziges Teilchen sich befindet, während alle im Innern des Kristalles angehäuft sind. Umgekehrt zeigt Tafel VII, Fig. $b$ eine eigentümlich netzartige Anordnung der Teilchen. Endlich kann es vorkommen, daB die Teilchen sich genau nach Kristallflächen anordnen, insbesondere in verzwillingten Kristallindividuen. Eine derartige Anordnung kann man z. B. in Tafel VII, Fig. 7, 8 und 9 erkennen. Die Teilchen sind in Fig. 7 hier stufenweise nach (001) gelagert. Die Streifensysteme der beiden nach (012) verzwillingten Individuen in Fig. 8 und 9 bilden untereinander einen Winkel von etwa $130^{\circ}$, dessen Halbierende die Spur der Zwillingsebene ist. Ähnliche Erscheinungen sind bisher nur bei Gaseinschlüssen in vulkanischen Mineralien (vor allen Plagioklasen und Augiten) beobachtet worden, an kolloidalen Teilchen unseres Wissens bisher nicht. Gaseinschlüsse finden sich übrigens in unseren Präparaten ebenfalls gelegentlich vor, doch sind dieselben ohne Schwierigkeit, schon wegen ihrer Größe, ihrer scharfen Einstellbarkeit und ihrer blendenden Lichtfülle von den kolloidalen Teilchen zu unterscheiden (Tafel VII, Fig. 10).

\section{Nachweise zur Natur der Metallnebel.}

Stellt man sich auf den Standpunkt, daß die Nebel, welche durch Zusatz von Blei zu geschmolzenem Bleichlorid auftreten, metallischer Natur sind (Metallnebel), so sind die vorbeschriebenen Erscheinungen leicht $z u$ verstehen. Beim Erkalten werden die feinen, in der Schmelze schwebenden Metalltröpfchen in die Kristallstruktur eingelagert, und das entstehende Produkt gleicht den von ZsiamoNDY beobachteten Rubingläsern, in denen metallisches Gold angenommen wird. Wir haben uns weiter davon überzeugt, daB auch die auf chemischem Wege hergestellten Metallnebel ${ }^{1}$ dasselbe ultramikroskopische Bild liefern wie die bisher besprochenen. $\mathrm{Zu}$ diesem Zwecke wurden vollkommen reine und optisch leere Bleichloridschmelzen im geschmolzenen Zustand mit einer Spur von Kaliumcyanid versetzt, wobei eine lebhafte Reaktion und Gasentwickelung beobachtet wird, deren Resultat die Schwarzfärbung der Schmelze ist. Die daraus erhaltenen Kristalle zeigen im Ultra-

1 R. Lonenz, G. von Hevesy und E. Wouff, Beiträge zur Kenntnis der Natur der Metallnebel in Schmelzflüssen. Zeitschr. phys. Chem. 76 (1911), 732. 
mikroskop dieselben Erscheinungen wie oben beschrieben, nur enthalten sie Gaseinschlüsse und kolloidale Teilchen nebeneinander (siehe Tafel VIII, Fig. 11). Bei Zusatz von etwas mehr Kaliumcyanid erhält man Kristalle, welche mit zahllosen Gasblasen durchsetzt sind und die kolloidalen Teilchen in weit gröBerer Konzentration entbalten, als dies durch direktes ,Nebeln" mittels Blei bei kürzerer Einwirkung erhältlich ist (siehe Tafel VIII, Fig. 12). Beim Wiederaufschmelzen der metallnebelhaltigen Kristalle erhält man unabhängig von ihrer Erzeugungsweise höchst intensiv gefärbte SchmelzHüsse wieder. Diese lassen sich dann durch Behandlung mit dem erwähnten Gemisch von Chlor- und Chlorwasserstoffgas wieder wasserklar machen, und es läBt sich beweisen, daB nach genügend langer Einwirkung die daraus entstehenden Kristalle optisch loer werden (siehe Tafel VIII, Fig. 13). Diese Operationen lassen sich beliebig oft in dem einen oder anderen Sinne wiederholen. Hierbei zeigt sich das Ultramikroskop von außerordentlicher Empfindlichkeit beim Nachweis solcher Nebelteilchen. Denn beim bloßen Wiederaufschmelzen einer optisch leeren Kristallmasse und Wiedererstarrenlassen derselben treten im Beleuchtungskegel sofort wieder kolloidale Teilchen auf (s. Tafel VIII, Fig. 14). Aus diesem Grunde ist auch käufliches Bleichlorid, wenn es auch als ,chemisch rein" bezeichnet ist, nach dem Umschmelzen niemals optisch leer. Diese Beobachtungen sprechen außerordentlich zugunsten der von R. LoRENz ${ }^{1}$ ausgesprochenen Vermutung, daß in den Metallhalogeniden sowohl im festen wie auch im flüssigen Zustand ein Dissoziationsgleichgewicht besteht, das durch die Formel

$$
\mathrm{n} \mathrm{MCl}_{2}=\underbrace{(\mathrm{n}-1) \mathrm{MCl}_{2}+\mathrm{M}}_{\text {genebelte Schmelze. }}+\mathrm{Cl}_{2},
$$

ausgedrückt werden kann. Der optisch leere Kristall ist eine Substanz, die sich bezüglich der Metallnebel im metastabilen Zustande befindet. Für diese Auffassung spricht auch die oben S. 48 bereits erwähnte Tatsache, daß optisch leere Kristalle nur erhalten werden, wenn man die „reinen" Schmelzen nicht zu langsam abkühlt. Ferner läBt sich beobachten, daß das Innerste von Schmelzflüssen, welche optisch leere Kristalle liefern, stets noch wenige kolloidale Teilchen aufweist, weil hier die Erstarrung zuletzt vor sich ging. Da der innere Kern am längsten flüssig war, so konnte sich in dieser Zeit das Gleichgewicht bereits wieder ausbilden.

1 Vgl. Elektrolyse der geschmolzenen Salze, Bd. 2 (Halle 1905), S. 64. 


\section{Zusammenfassung.}

1. Reines, optisch vollkommen leeres Bleichlorid wurde in größeren Kristallen aus dem Schmelztluß dargestellt.

2. Einige physikalische und kristallographische Eigenschaften dieser Kristalle wurden untersucht.

3. Schmelzen von optisch leerem Bleichlorid wurden durch Zusammenschmelzen mit metallischem Blei mit Bleinebeln durchsetzt, und der Vorgang der Nebelbildung wurde photographiert. Metallnebelhaltige Kristalle wurden dargestellt.

4. Kristalle von reinem und von mit Metallnebeln erfülltem Bleichlorid wurden ultramikroskopisch untersucht und die kolloidale, den Rubingläsern analoge Natur der metallnebelhaltigen Kristalle festgestellt.

5. Es wurden metallnebelhaltige Kristalle in optisch leere verwandelt und nachgewiesen, daB sich die beidersinnigen Umwandlungen beliebig oft reproduzieren lassen.

6. Ferner wurde der ultramikroskopische Nachweis geführt, daB der auf chemischem Wege (durch Kaliumcyanid) entstehende Metallnebel in den Kristallen identisch ist mit dem aus metallischem Blei gewonnenen.

Frankfurt a. M., Institut für physikalische Chemie der Universität und des Physikalischen Vereins.

Bei der Redaktion eingegangen am 28. September 1914. 